ISEF 2007 - XIII International Symposium on Electromagnetic Fields

in Mechatronics, Electrical and Electronic Engineering

Prague, Czech Republic, September 13-15, 2007

\title{
OPTIMISATION OF A DRIVE SYSTEM AND ITS EPICYCLIC GEAR SET
}

\author{
Nicolas BELLEGARDE, Philippe DESSANTE, Pierre VIDAL, Jean-Claude VANNIER
}

Département Énergie - Supélec, 1 rue Joliot-Curie 91192 Gif-sur-Yvette, France, nicolas.bellegarde@supelec.fr

\begin{abstract}
This paper describes the design of a drive consisting of a DC motor, a speed reducer, a lead screw transformation system, a power converter and its associated DC source. The objective is to reduce the mass of the system. Indeed, the volume and weight optimisation of an electrical drive is an important issue for embedded applications. Here, we present an analytical model of the system in a specific application and afterwards an optimisation of the motor and speed reducer main dimensions and the battery voltage in order to reduce the weight.
\end{abstract}

\section{INTRODUCTION}

The system studied in this paper is a linear electrical drive system realized with a Ni-MH battery bank, a DC/DC converter, a DC motor, a speed reducer and a lead-screw device. The aim of the system is to move a load along a linear displacement. Regarding the load, we can define mainly two specifications. Firstly, it has to apply a rather high static force to overcome some static friction force. This has to be done at constant speed or at standstill. Secondly, it has to be driven from one point to another in a given time. This specification implies a dynamic force, a given acceleration and a maximum speed depending on the kind of displacement.

In order to optimise the weight of the system, and mainly the battery, DC motor and speed reducer weights, geometrical and physical relations have to be written for each component. These relations are then linked with the others to make a global optimisation of the system. The constraints are based upon the load specifications. The mathematical optimisation is performed with the help of various numerical methods like Genetic Algorithm, Random Search, Differential Evolution and Nelder Mead.

\section{SYSTEM MODEL}

\section{Power conversion system}

A general representation of the system is given in figure 1.

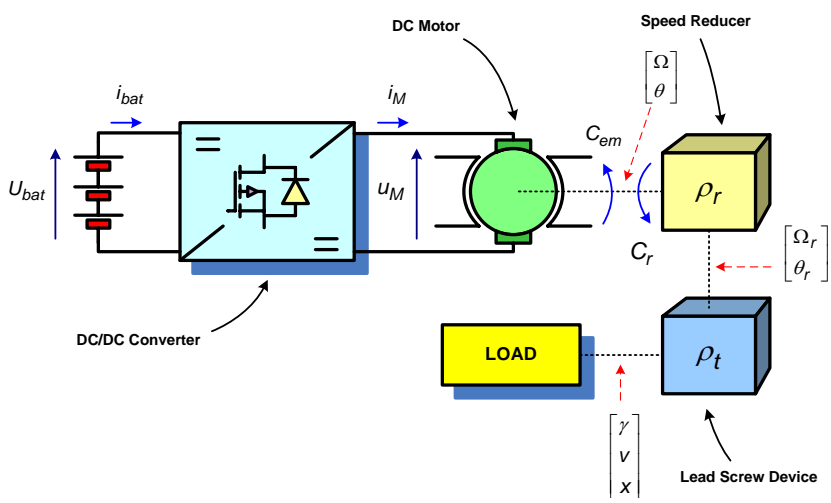

Figure 1: Power conversion system.
$C_{e m}:$ torque developed by the motor [ $N$ ]

$C_{r}$ : resistive torque [ $\left.N\right]$.

$\rho_{r}:$ transformation ratio of the speed reducer.

$\rho_{t}:$ transformation ratio of the lead-screw device $[\mathrm{rad} / \mathrm{m}]$.

$\theta_{m}$ : motor shaft angle [ rad ].

$\Omega_{m}$ : motor shaft speed [ $\mathrm{rad} / \mathrm{s}$ ].

$x$ : linear displacement $[m]$.

$v$ : linear speed $[\mathrm{m} / \mathrm{s}]$. 
Concerning the mechanical part, the lead-screw is represented by its transformation ratio deduced from the screw pitch while the speed reduction system introduces a speed transformation ratio.

The relation between the motor shaft angle $\theta_{m}$ and the linear displacement $x$ can be written as follows:

$$
\theta_{m}=\rho_{r} \rho_{t} \cdot x=\rho \cdot x
$$

Where $\rho$ is the global transformation ratio.

\section{Speed reducer volume}

A representation of the speed reducer is given in figure 2 .

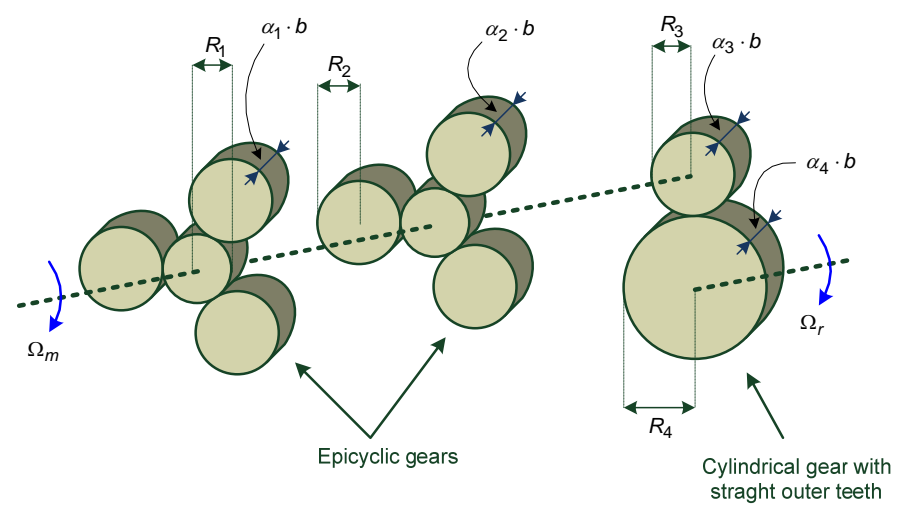

Figure 2: Speed reducer structure.

The speed reducer is constitued by two epicyclic gears (characterized by $R_{1}, R_{2}$ ) and one cylindrical gear with straght outer teeth (characterized by $R_{3}, R_{4}$ ). We can express the volume $V_{r}$ of this speed reducer as follows:

$$
V_{r}=\pi \cdot b \cdot\left[\left[R_{1}+2 \cdot R_{2}\right]^{2} \cdot\left[\alpha_{1}+\alpha_{2}\right]+R_{3}{ }^{2} \cdot \alpha_{3}+R_{4}{ }^{2} \cdot \alpha_{4}\right]
$$

\section{Load specifications}

Here, all load specifications are expressed on the motor shaft. In the considered application, the motor has to generate two sorts of torques, imposed by the load. A static torque which is necessary to reach the breakaway force on the load just before it starts to move or to maintain the speed at a constant value. The static torque $C_{e m_{s}}$ is given by:

$$
C_{e m_{s}}=K_{1} \cdot \frac{R_{3}}{\left[1+\frac{R_{2}}{R_{1}}\right]^{2} \cdot R_{4}}
$$

Where $K_{1}$ is a constant depending on the speed, the lead-screw transformation ratio, the different resistive forces due to the load.

The motor must also generate a dynamic torque which is required when the different resistive forces are at their maximum values. 
The dynamic torque $C_{e m_{d}}$ can be expressed as follows:

$$
C_{e m_{d}}=K_{2} \cdot \frac{R_{3}}{\left[1+\frac{R_{2}}{R_{1}}\right]^{2} \cdot R_{4}}
$$

Where $K_{2}$ is a constant depending on the same parameters as $K_{1}$.

An other torque can be also considered. Indeed, the RMS torque $C_{e m_{R M S}}$ is interesting because it is directly linked to the copper losses in the motor. Its expression is given by:

$$
C_{e m_{R M S}}{ }^{2}=K_{3} \cdot J^{2} \cdot \frac{\left[1+\frac{R_{2}}{R_{1}}\right]^{4} \cdot R_{4}{ }^{2}}{R_{3}{ }^{2}}+K_{4} \cdot \frac{R_{3}{ }^{2}}{\left[1+\frac{R_{2}}{R_{1}}\right]^{4} \cdot R_{4}{ }^{2}}+K_{5} \cdot J
$$

$\mathrm{J}$ is the total inertia of the system. The constants $K_{3}, K_{4}, K_{5}$ are functions of the average and the rms values of the load speed and acceleration, the lead-screw transformation ratio and the average and the rms values of the different resistive forces.

\section{$\underline{\text { 4. Motor specifications }}$}

Among the limits concerning the motor, there is a maximal rotor speed $\Omega_{M}$, function of the maximal linear speed $v_{M}$, defined by:

$$
\Omega_{M}=\rho_{r} \rho_{t} \cdot v_{M}
$$

The maximum peak power $P_{M}$ given by the motor to the load has also to be considered, where $K_{6}$ is a constant depending on the different resistive forces:

$$
P_{M}=K_{6} \cdot v_{M}
$$

For the motor's design, it is possible to define the main dimensions by the peak torque $C_{e m_{M}}$, the nominal torque $C_{e m_{n}}$ and the rotor inertia $J_{m}$ as seen in previous papers [1-6]:

$$
\left\{\begin{array}{l}
C_{e m_{n}}=2 \pi \cdot A_{L} \cdot B \cdot \gamma_{p} \cdot R^{2} \cdot L \\
C_{e m_{M}}=4 \cdot p \cdot H_{M} \cdot B \cdot R \cdot L \cdot E \\
J_{m}=\frac{\pi}{2} \cdot \mu_{m} \cdot R^{4} \cdot L
\end{array}\right.
$$

These three relationships introduce three main dimensions parameters for the design : the rotor radius $R$, the rotor length $L$ and the permanent magnet thickness $E$. The remaining parameters are constants and have the following significations:

- $\quad p=1$ : pole's number.

- $H_{M}=80 \mathrm{kA} / \mathrm{m}$ : magnet's peak magnetic field.

- $\quad B=0,4 T$ : air gap flux density.

- $A_{L}=7850 \mathrm{~A} / \mathrm{m}$ : rms current linear density.

- $\gamma_{p}=0,75$ : pole's overlapping factor.

- $\mu_{m}=5070 \mathrm{~kg} / \mathrm{m}^{3}$ : motor mass density 


\section{SYSTEM OPTIMISATION}

The established relationships are used to define the constraints during the optimisation procedure. Two types of constraints are considered: the physical constraints which permit to ensure that the motor can supply load requirements and the geometrical constraints which permit to define a feasible motor and speed reducer (see [7] for futher details).

Concerning the physical constraints, the motor peak torque has to be greater than the static and dynamic torques. The nominal torque has also to be greater than the required rms torque.

$$
\left\{\begin{array}{l}
C_{e m_{M}}>C_{e m_{d}} \\
C_{e m_{M}}>C_{e m_{s}} \\
C_{e m_{n}}>C_{e m_{R M S}}
\end{array}\right.
$$

The maximum power supplied by the battery bank has to be greater than the maximum power consumption.

$$
\left[U_{b a t} \cdot i_{b a t}\right]_{M}>P_{M}
$$

The maximum speed required by the load has to be kept lower than the nominal speed of the motor $\Omega_{n}$.

$$
\Omega_{n}>\rho_{r} \rho_{t} \cdot v_{M}
$$

Concerning the geometrical constraints, the following will be used in order to have feasible motor and speed reducer:

$$
\left\{\begin{array}{l}
R \geq 4 \mathrm{~mm} \\
R \leq L \leq 5 \cdot R \\
R \geq E \geq 3 \mathrm{~mm} \\
R_{2} \geq R_{1} \geq 4 \mathrm{~mm} \\
R_{4} \geq R_{3} \geq 6 \mathrm{~mm}
\end{array}\right.
$$

Depending on the application, different cost functions can be minimized. Here, we present an optimisation where the weight of the system is the cost function. We sum the motor, speed reducer and battery bank masses:

$$
M=M_{\text {bat }}+M_{m}+M_{r}
$$

The motor and speed reducer masses are defined as follows:

$$
\begin{gathered}
M_{m}=\mu_{m} \cdot \pi \cdot R^{2} \cdot L \\
M_{r}=\pi \cdot \mu_{r} \cdot b \cdot\left[\left[R_{1}+2 \cdot R_{2}\right]^{2} \cdot\left[\alpha_{1}+\alpha_{2}\right]+R_{3}{ }^{2} \cdot \alpha_{3}+R_{4}{ }^{2} \cdot \alpha_{4}\right]
\end{gathered}
$$

The battery bank mass is a function of the voltage if we take a constant capacity.

$$
M_{\text {bat }}=f\left(U_{\text {bat }}\right)
$$


The total weight can be expressed by the following expression:

$$
M=f\left(U_{b a t}\right)+\mu_{m} \cdot \pi \cdot R^{2} \cdot L+\pi \cdot \mu_{r} \cdot b \cdot\left[\left[R_{1}+2 \cdot R_{2}\right]^{2} \cdot\left[\alpha_{1}+\alpha_{2}\right]+R_{3}{ }^{2} \cdot \alpha_{3}+R_{4}{ }^{2} \cdot \alpha_{4}\right]
$$

The seven optimisation parameters are: $R, L, E, R_{1}, R_{2}, R_{3}, R_{4}$.

\section{IV.RESULTS}

We present here the results concerning the definition of the battery, the speed reducer and the motor whose characteristics are optimised for a given load. In this example, the load characteristics are presented in the table I.

\begin{tabular}{|c|c|}
\hline$\gamma_{\text {eff }}=30,49 \mathrm{~mm} / \mathrm{s}^{2}$ & $v_{\text {eff }}=12,42 \mathrm{~mm} / \mathrm{s}$ \\
\hline$\gamma_{M}=60 \mathrm{~mm} / \mathrm{s}^{2}$ & $v_{M}=15 \mathrm{~mm} / \mathrm{s}$ \\
\hline$K_{1}=0,23 \mathrm{~N}$ & $K_{2}=4,47 \mathrm{~N}$ \\
\hline$K_{3}=23496 \mathrm{~N}^{2} / \mathrm{kg}{ }^{2} \cdot \mathrm{m}^{4}$ & $K_{4}=3,46 \mathrm{~N}^{2}$ \\
\hline$K_{5}=-140,4 \mathrm{~N}^{2} / \mathrm{kg} \cdot \mathrm{m}^{2}$ & $K_{6}=14995 \mathrm{~W} \cdot \mathrm{s} / \mathrm{m}$ \\
\hline
\end{tabular}

Table I : Load characteristics.

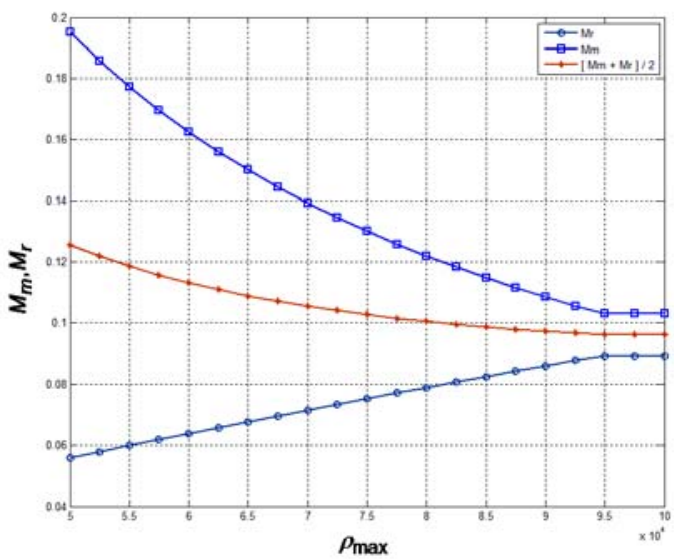

Figure 3: Motor and speed reducer weights versus $\rho_{\max }$.

Before optimisation, the battery voltage is $14 \mathrm{~V}$ and the weight $M$ is equal to $1180 \mathrm{~g}$. The optimisation procedures uses the constraints (9-12) and searches a set of values for $R, L, E, R_{1}, R_{2}, R_{3}, R_{4}$ and $U_{b a t}$ which minimises the total mass of the system in equation (17).

The optimisation is made with Mathematica and verfied with Matlab and an home made genetic algorithm.

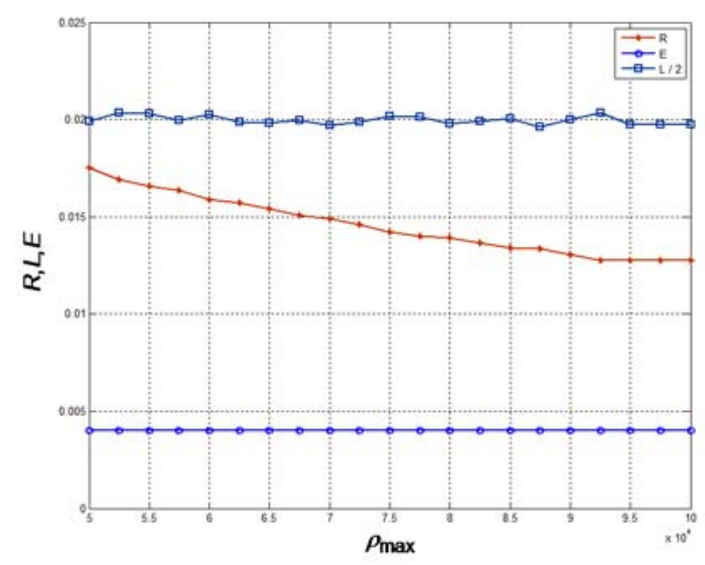

Figure 4: Motor dimensions versus $\rho_{\max }$.

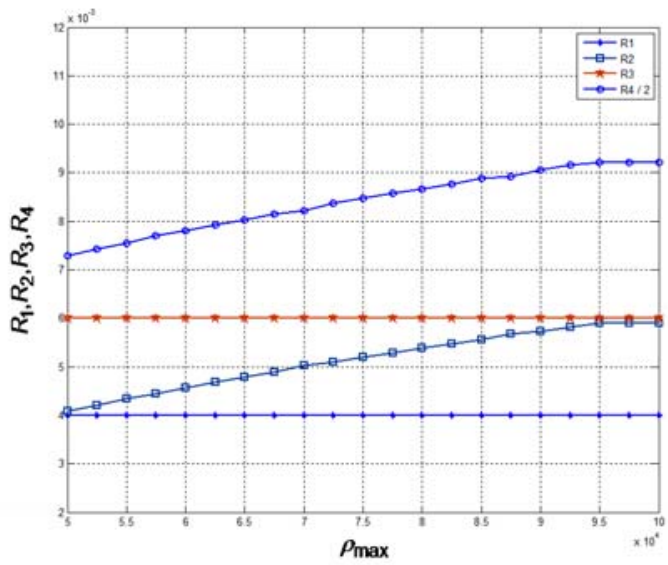

Figure 5: Speed reducer dimensions versus $\rho_{\max }$. 
Figures 3-5 show the results obtained after the optimisation procedure. The battery voltage is equal to $12 \mathrm{~V}$ and its weight is $640 \mathrm{~g}$.

As seen in a previous paper [4-5], boundaries are used to limit the variation of the parameters to feasible values. It appears that the results are controlled by the upper boundary value for the transformation ratio $\rho=\rho_{r} \rho_{t}$.

Figure 3 shows the motor and speed reducer weights of the system in function of the constraint $\rho_{\max }$. The system mass decreases until $\rho_{\max }=95 \mathrm{rad} / \mathrm{mm}$, where it remains constant. Before the previous value, the minimum is obtained for $\rho=\rho_{\max }$. There is no need to have a transformation ratio greater than $95 \mathrm{rad} / \mathrm{mm}$ because the system weight will not decrease anymore. At this point, the decrease of the motor weight is compensated by the increase of the speed reducer weight. The resulting active mass is $830 \mathrm{~g}$.

Figures 4 and 5 show the dimensions of the motor and the speed reducer versus the upper constraint $\rho_{\max }$. Concerning the motor, we can see in Figure 4 that the radius decreases until $\rho_{\max }=95 \mathrm{rad} / \mathrm{mm}$ and the two others parameters are independant of $\rho_{\max }$. In figure 5 , we can note that two radius increase until $\rho_{\max }=95 \mathrm{rad} / \mathrm{mm}$ while the two others stay constant.

\section{V.CONCLUSION}

In this paper, the weight of an electromechanical conversion system has been optimised. Firstly, a model of the motor and the speed reducer has been done. This model links the motor and speed reducer main dimensions to their performances. Then, the battery bank, motor and speed reducer weights have been written in function of the optimisation parameters. Secondly, an optimisation procedure was executed in order to minimise the objective function which is the weight of the previous components. Different numerical optimisation methods were used to valid the methodology. All gave the same results. The initial total weight was $1180 \mathrm{~g}$ and the obtained total weight after optimisation is equal to $830 \mathrm{~g}$. We can note that the weight is reduced by $30 \%$.

\section{ACKNOWLEDGMENT}

The authors would like to thank Virax company, and particularly Mr. Frédéric BERNIER for his help and his financing.

\section{$\underline{\text { References }}$}

[1] Macua E., Ripoll C., Vannier J-C, Design and Simulation of a Linear Actuator for Direct Drive, PCIM2001, pp 317-322, june 19-21, 2001, Nürnberg (Germany).

[2] Macua E., Ripoll C., Vannier J-C, Design, Simulation and Testing of a PM Linear Actuator for a Variable Load, PCIM2002, pp 55-60, may 14-16, 2002, Nürnberg (Germany).

[3] Macua E., Ripoll C., Vannier J-C, Optimization of a Brushless DC Motor Load Association, EPE2003, Sept 0204, 2003, Toulouse (France).

[4] Ph. Dessante, J-C Vannier, Ch. Ripoll, Optimisation of a Linear Brushless DC Motor Drive, ICEM 2004, September 2004.

[5] Ph. Dessante, J-C Vannier, P. Vidal, Optimisation of a Linear Brushless DC Motor Drive and the Associated Power Supply, AES 2005 Civil or Military All-Electric Ship Conference, 13 - 14 October 2005 ParisVersailles, France.

[6] Rioux C., Théorie générale comparative des machines électriques établie à partir des équations du champ électromagnétique, Revue générale de l’Electricité (RGE), mai 1970.

[7] Nurdin M., Poloujadoff M., Faure A., Synthesis of Squirrel Cage Motor : A Key to Optimisation, IEEE Trans on Energy Conversion, Feb 1994. 